\title{
THE COMPOSITION OF DUST IN STELLAR EJECTA
}

\author{
D. C. B. WhitTeT* \\ Lancashire Polytechnic, Preston, UK
}

\begin{abstract}
I review the observational evidence relevant to the composition of dust ejected from circumstellar shells. Emphasis is placed on those types of stars believed to be the principal sources of refractory stardust in the interstellar medium. In stars with normal abundances $(O>C)$, the predominant species appears to be an amorphous silicate, characterised by smooth spectral features at 9.7 and $19 \mu \mathrm{m}$, a far infrared emissivity close to unity, and a featureless ultraviolet extinction curve. Similarly, the characteristics of carbon stars $(C>O)$ are consistent with the presence of amorphous carbon rather than graphite. Silicon carbide is a minor constituent of carbon-rich stardust and is therefore unlikely to be an important ingredient of interstellar grains. A major goal for future studies will be to establish the evolutionary link between amorphous carbon stardust and the carriers of the unidentified infrared emission features observed in planetary nebulae.
\end{abstract}

\section{INTRODUCTION}

Stars may be associated intimately with dust at almost any phase of their evolution. Starbirth currently occurs in dusty molecular clouds in the disc of our Galaxy, and the dust grains appear to play a vital role in maintaining the thermal balance of newly formed stars during the initial stages of their evolution. Much of the placental grain material may be returned subsequently to the interstellar medium, but, in some cases, remnant protostellar discs or shells survive well into the main sequence phase. Star formation undoubtedly influences the dust content of the local interstellar medium in which it occurs, either by direct recycling or by radiative dissipation and processing. However, it is not until the post-main-sequence phase is reached that stars appear to become significant sources of new grain material in the interstellar medium (Bode, 1988; Gehrz, 1989).

The cool atmospheres of late-type giants and supergiants provide an ideal environment for the nucleation and growth of refractory particles. A detailed discussion of models for grain nucleation and growth in stellar atmospheres may be found elsewhere in this Symposium (Sedlmayr, 1989) and in the classic review paper of Salpeter (1977). For the purposes of the discussion presented here, we merely note that the composition of solid condensates is expected to be governed by the gas phase $C / O$ abundance ratio. In stars with 'normal' abundances (i.e. $O>C$, as in the Sun), much of the available $C$ is tied up in gas phase $C O$, leaving excess

*currently on leave at York University and CITA (University of Toronto), Ontario, Canada. 
$O$ to combine with heavier elements in solid particles. Conversely, in stars where $C$-enrichment has occurred to the extent that $C>O$, the roles are reversed, with $O$ tied up in gas phase $C O$ and $C$ free to condense into solids.

The presence of dust around stars is demonstrated observationally in a number of ways; phenomena attributed to circumstellar dust include:

(a) reflection nebulae;

(b) infrared continuum emission;

(c) dust-related spectral features; and

(d) circumstellar extinction.

All of these phenomena can, in principle, give information relevant to grain composition, and the latter three are discussed in detail in the following sections. Observations of reflection nebulae associated with young, early-type stars have been used to quantify the albedo and phase function of the grains (e.g. Witt, 1985), but no corresponding studies of evolved objects have been attempted to date. Indeed, there are few cases where visible nebulosity can be attributed with confidence to scattering by dust originating in the atmosphere of the illuminating star.

A promising example of an apparently self-generated reflection nebula is illustrated in Color Plate 5. The nebula IC2220 surrounds the bright star HR3126, an otherwise normal red giant (spectral type MOIII) situated approximately 110pc from the Sun in a direction of low general galactic extinction. An infrared excess in the star's broadband flux distribution (Humphreys and Ney, 1973) confirms the presence of locally heated dust. The dimensions of the nebula ( $\sim 3$ arcmin) suggest an expansion time of $\sim 10^{4}$ years at a velocity of $10 \mathrm{~km} \mathrm{~s}^{-1}$, characteristic of red giant winds, which is compatible with the likely post-main-sequence age of the star. This object is highlighted for future study, as it provides a rare opportunity to investigate circumstellar extinction, scattering, and infrared emission in the same line of sight.

In the preceding paper, Gehrz has identified the principal sources of ejected stardust in the interstellar medium, and estimated their production rates. In the remainder of this review, I examine the observational evidence relating specifically to grain composition. The order of discussion is dictated by observational phenomena, as itemised above, rather than by object type. For more specific discussion of dust in planetary nebulae and in supernovae, see the recent reviews by Roche (1988) and Dwek (1989), respectively; for a general review of dust in circumstellar shells, see Bode (1988).

\section{INFRARED CONTINUUM EMISSION}

Infrared emission greatly in excess of that from a normal photosphere is a definitive characteristic of stars with dust shells. Luminous energy from the photosphere is absorbed by the grains and re-emitted at lower frequencies. The emergent flux density is

$$
F_{\nu} \propto Q_{F I R} B\left(\nu, T_{g}\right),
$$

where $Q_{F I R}$ is the far infrared emissivity of the grain material, $T_{g}$ the grain temperature, and $B$ the Planck function (see Hildebrand, 1983). In the Rayleigh-Jeans 


\section{TABLE 1}

Circumstellar infrared features observed in evolved stars

\begin{tabular}{|c|c|c|c|c|c|}
\hline$\lambda(\mu \mathrm{m})$ & Carrier & Abs/em? & ISM? & C/O? & Object type \\
\hline $\begin{array}{l}3.1,6.0 \\
11.5\end{array}$ & $\begin{array}{l}\mathrm{H}_{2} \mathrm{O} \text { ice } \\
\mathrm{H}_{2} \mathrm{O} \text { ice }\end{array}$ & $\begin{array}{l}\mathbf{a} \\
\mathbf{a}\end{array}$ & $\begin{array}{l}\text { Yes } \\
\text { No }\end{array}$ & $\begin{array}{l}O>C \\
O>C\end{array}$ & $\begin{array}{l}\text { OH-IR/post-AGB stars } \\
\text { As above }\end{array}$ \\
\hline $\begin{array}{l}3.3,3.4 \\
6.2,7.7 \\
8.6,11.3\end{array}$ & PAHs? & $\mathbf{e}$ & Yes & $C>O$ & PN, proto-PN \\
\hline $\begin{array}{l}9.7,19 \\
11.2 \\
30\end{array}$ & $\begin{array}{l}\text { Silicates } \\
S i C \\
M g S ?\end{array}$ & $\begin{array}{l}\mathbf{a} / \mathbf{e} \\
\mathbf{e}\end{array}$ & $\begin{array}{l}\text { Yes } \\
\text { No } \\
\text { No }\end{array}$ & $\begin{array}{l}O>C \\
C>O \\
C>O\end{array}$ & $\begin{array}{l}\mathrm{K}, \mathrm{M} \text { stars, PN } \\
\text { Carbon stars, PN } \\
\text { Extreme C-stars, PN }\end{array}$ \\
\hline
\end{tabular}

approximation,

$$
F_{\nu} \propto \nu^{\beta+2}
$$

where $\beta$ is the spectral index of the emissivity (i. e. $Q_{F I R} \propto \nu^{\beta} \propto \lambda^{-\beta}$ ). Thus $\beta$ can, in principle, be determined from the slope of the far infrared flux distribution. This is relevant to the composition because different values of $\beta$ are predicted by different models (see Whittet, 1988 for a brief review). In particular, $\beta \simeq 1$ for amorphous materials and $\beta \simeq 2$ for crystalline materials.

Far infrared data are now available for a number of cool, dust-ejecting stars. The well known carbon star IRC+10216 is perhaps the best studied of all such objects (e.g. Sopka et al., 1985; Le Bertre, 1987; Martin and Rogers, 1987). Figure 1 shows the spectral energy distribution for IRC+10216 and the similar but more highly obscured carbon star CRL3068, both of which are well-fitted by a model with $\beta=1.2 \pm 0.2$. These data lead Sopka et al. to conclude that the dust in the vicinity of these objects is characterised by an emissivity variation that suggests amorphous carbon rather than crystalline graphite. More generally, $\beta$ values close to unity are typical of dusty late-type stars irrespective of $C / O$ ratio (Sopka et al., 1985; Rowan-Robinson et al., 1985), consistent with amorphous grain structure in both $C$-rich and $O$-rich environments.

\section{INFRARED SPECTRAL FEATURES}

Table 1 lists the dust-related spectral features observed in circumstellar environments around evolved stars. The columns indicate: (i) the wavelength in microns; (ii) the proposed carrier; (iii) an indication of whether the feature is seen in absorption or emission; (iv) whether the feature has a counterpart in the interstellar medium; (v) the $\mathrm{C} / \mathrm{O}$ ratio of the source; and (vi) the object type. This list of features may conveniently be divided into three groups according to carrier type: 


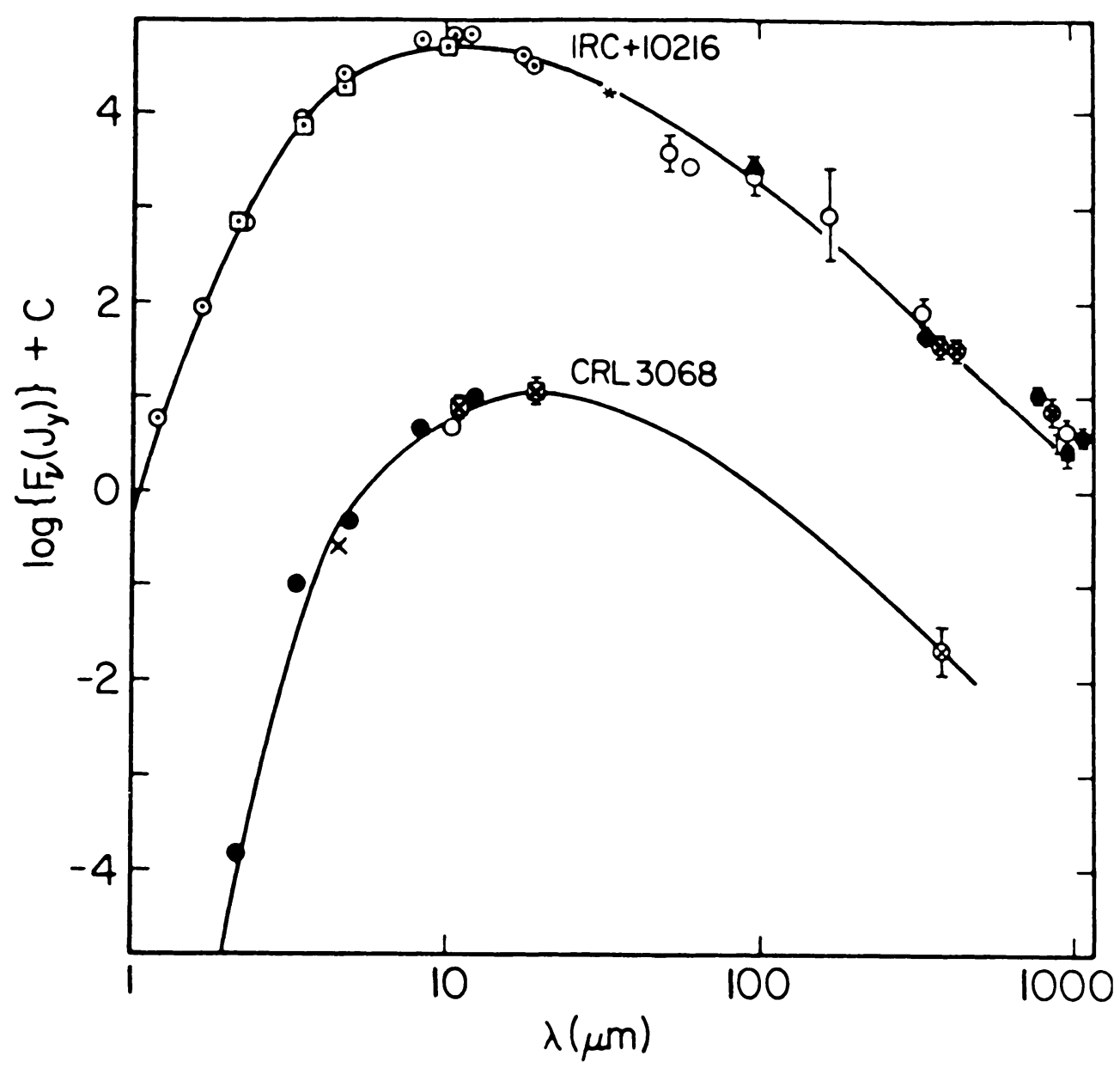

Fig. 1. Spectral energy distributions in the infrared for carbon stars IRC+10216 and CRL3068. The symbols represent observational data from various authors, the curves are based on an amorphous carbon grain model assuming emissivity $Q_{F I R} \propto \lambda^{-1.2}$. (See Sopka et al., 1985 for further details). 
1. those associated with relatively refractory materials ('stardust'), i.e. silicates, $S i C$ and $M g S$;

2. the 'unidentified' infrared emission features, usually associated with polycyclic aromatic hydrocarbons (PAHs), of which only the principal wavelengths are listed in Table 1; and

3. those identified with water-ice.

These groups are discussed individually below.

\subsection{STARDUST}

The occurrence of spectral features near 10 and $20 \mu \mathrm{m}$ associated with dust in evolved stars has been known for a number of years (e.g. Treffers and Cohen, 1974). The $11.2 \mu \mathrm{m}$ silicon carbide feature is seen in stars classified optically as carbon-rich, whilst the 9.7 and $19 \mu \mathrm{m}$ features, associated with stretching and bending modes in silicates, are seen in $M$ stars. A few exceptional cases have been found in which optically-classified carbon stars show silicate features: these may perhaps be representative of binary systems with both $O$-rich and $C$-rich components (Bode, 1988 and references therein). In general, the correlation between optical spectral type and infrared dust spectrum is extremely strong, and it is thus reasonable to use the latter as a diagnostic of $C / O$ abundance ratio in stars too heavily obscured to be studied optically. This situation also holds for planetary nebulae (see Roche, 1988).

The position, width, and profile shape of the infrared features can provide information on grain size and structure. Average profiles of the $11.2 \mu \mathrm{m} \mathrm{SiC}$ and $9.7 \mu \mathrm{m}$ silicate features in red giants are illustrated in Figure 2, which is based on low resolution spectra (LRS) from the IRAS catalog (Little-Marenin, 1986). The $\mathrm{SiC}$ profile appears to be relatively constant from star to star (but see also Cohen, 1984). The $9.7 \mu \mathrm{m}$ silicate feature is more difficult to study because of uncertainties in the continuum level. In addition, the short wavelength cutoff of the LRS at $7.5 \mu \mathrm{m}$ is close to the edge of the feature. However, real variations in width are apparent. The relatively narrow feature observed in some late-type stars (e.g. $\mu$ Cephei) agrees well with that seen in absorption in the diffuse interstellar medium (Roche and Aitken, 1984a), suggestive of a compositional/mineralogical similarity between circumstellar and interstellar grains. In other stars, broader $9.7 \mu \mathrm{m}$ profiles more characteristic of the Trapezium emissivity have been reported (Simpson and Rubin, 1989). It is probable that these variations are related to the thermal history of the grains (Nuth et al., 1986) and/or grain size effects (Papoular and Pegourie, 1983), although it is surprising that no corresponding variations are apparent in the $19 \mu \mathrm{m}$ feature (Simpson and Rubin, 1989). What is most striking is the general absence of fine structure in the observed 9.7 and $19 \mu \mathrm{m}$ profiles in many lines of sight, a result which is consistent with amorphous rather than crystalline grain structure.

The dust ejected from novae is generally presumed to be carbon-rich (e.g. Bode, 1988). It is therefore remarkable that two recent dusty novae with well-studied infrared spectra show evidence for silicate emission. QU Vul has been described in detail by Gehrz et al. (1986). The spectrum of V1370 Aql is illustrated in Figure 3. The prominent emission feature around $10 \mu \mathrm{m}$ strongly resembles the silicate feature, 

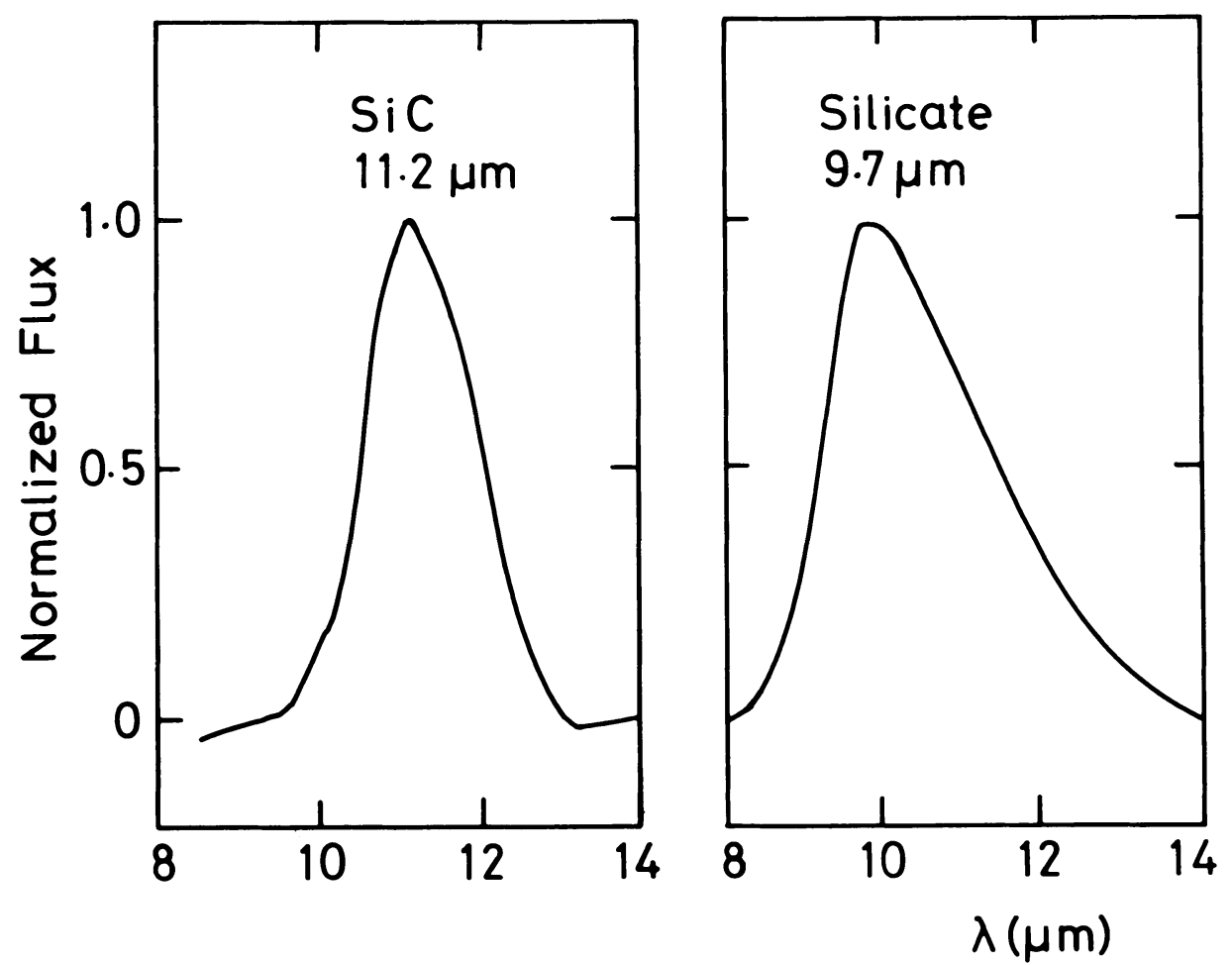

Fig. 2. Smoothed mean profiles of the $11.2 \mu \mathrm{m}$ silicon carbide and $9.7 \mu \mathrm{m}$ silicate features in carbon stars and M-type stars, respectively, based on IRAS low resolution spectra. The FWHM values are 1.6 and $2.4 \mu \mathrm{m}$.

although the apparent absence of a corresponding excess at $19 \mu \mathrm{m}$ led Gehrz et al. (1984) to suggest that the $10 \mu \mathrm{m}$ emission arises in a modified form of $S i C$-rich dust. However, the depletions of gas phase elements in V1370 Aql support the hypothesis that both $O$-rich and $C$-rich material has condensed in its outflow (Snijders et al., 1987).

A broad feature centred near $30 \mu \mathrm{m}$ has been observed in a small sample of $C$ rich planetary nebulae and extreme carbon stars, and attributed to $M g S$, possibly as a mantle on more refractory grain cores (Goebel and Mosely, 1985; Roche, 1988). $M g S$ is unlikely to be a major component of the dust ejected from these objects and cannot therefore be a significant component of interstellar dust, although its abundance may in some cases exceed that of $S i C$ (Martin and Rogers, 1987).

A narrow spectral feature predicted at $11.52 \mu \mathrm{m}$ may be used as a diagnostic for the presence of graphite in circumstellar shells (Draine, 1984). The non-detection of this feature in Ve2-45 (Glasse et al., 1986) and IRC+10216 (Martin and Rogers, 1987) leads to the conclusion that no more than about 3-5 percent of the solid carbon in the shells of these objects is graphitic. It would be worthwhile to extend this search to other objects. 


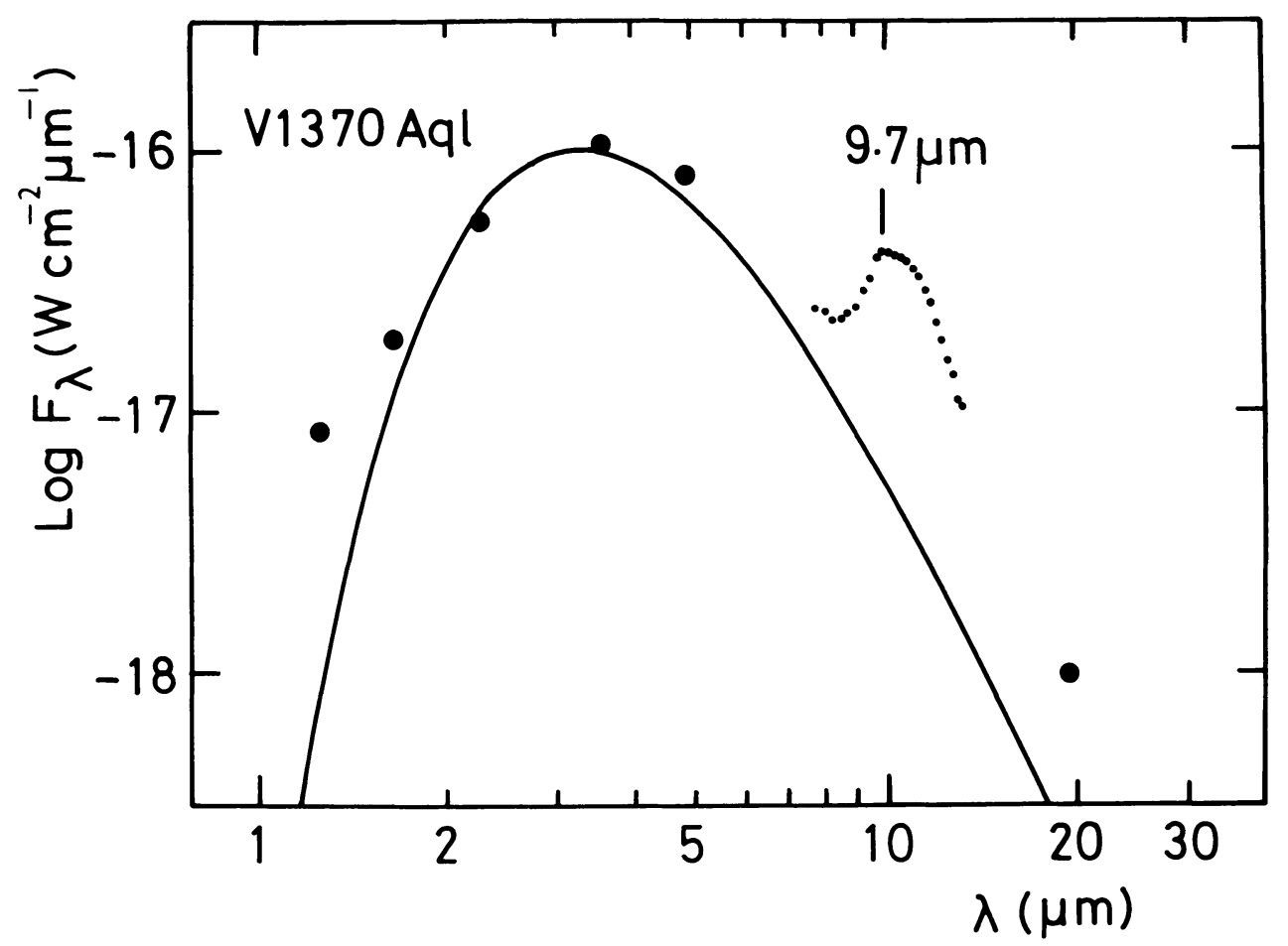

Fig. 3. Spectral energy distribution for Nova Aquilae 1982 (V1370 Aql) on day 156, based on broadband photometry (large points) and $8-14 \mu \mathrm{m}$ spectrophotometry (small points). A $T=620 \mathrm{~K}$ blackbody curve is also shown. (See Snijders et al., 1987).

\subsection{THE UNIDENTIFIED INFRARED FEATURES}

The UIR features, and their interpretation in terms of polycyclic aromatic hydrocarbons (PAHs), have been discussed at length elsewhere in this Symposium, and are considered only briefly here. A thorough account of their observed properties is given by Bregman (1989).

It is well-known that the UIR features are observed in a wide variety of environments, including reflection nebulae, HII regions and active galactic nuclei. However, in the context of stellar ejecta, they occur almost exclusively in the outflows of $C$-rich planetary nebulae and their precursors, with a strength that correlates significantly with $C / O$ ratio (Cohen et al., 1986; Roche, 1988). This clearly supports an origin in materials associated with carbonaceous stardust.

Types of PAH molecules which might account for the observed UIR spectrum are discussed by (e.g.) Allamandola, Tielens, and Barker (1987). Observational investigations of the relative strengths and profiles of the individual features in different sources may provide a discriminator between candidates. For example, recent spectroscopy at grating resolution has demonstrated variations in the strength of 
the $3.3 \mu \mathrm{m}$ feature relative to $3.4 \mu \mathrm{m}$ and other weaker features (de Muizon, Geballe and d'Hendecourt, 1986), and also in the $3.3 \mu \mathrm{m}$ profile itself (Tokunaga et al., $1988,1989)$. The observed profiles are, in turn, markedly different from those of typical laboratory PAHs, more closely resembling that of quenched carbonaceous composite. Much of the discussion at this Symposium has concerned matching the observations in other regions of the spectrum (e.g. the $11.3 \mu \mathrm{m}$ feature), but even at $3.3 \mu \mathrm{m}$, where there is good agreement between the observed and predicted positions of the feature for a given vibrational mode in PAHs, the detailed profile fits required in addition to wavelength coincidence for unique spectral identification of specific molecules have not yet been achieved.

\subsection{WATER-ICE}

Absorption features attributed to water frosts condensing in stellar outflows have been observed in the spectra of several OH-IR and post-AGB stars (e.g. Soifer et al., 1981; Eiora et al., 1983; Roche and Aitken, 1984b; Geballe et al., 1988). Although these objects are unlikely to be important sources of volatile grain material in the interstellar medium, their spectra are nevertheless of interest as a comparison for those of molecular cloud infrared sources.

Such a comparison is shown in Figure 4. Both spectra show deep ice absorption centred at $3.1 \mu \mathrm{m}$ (the $O-H$ stretch) and a weaker bending mode absorption at $6.0 \mu \mathrm{m}$. Both also show deep silicate absorption at $9.7 \mu \mathrm{m}$, but in the OH-IR star this is blended with a feature centred at $11.5 \mu \mathrm{m}$ and attributed to the water-ice libration mode. Other features present in the molecular cloud (at 3.4, 4.7 and $6.8 \mu \mathrm{m}$ ) are very weak or absent in the OH-IR star. This is consistent with current views on the composition of ices in molecular clouds (e.g. Whittet, 1988 and references therein): the carriers of these features are more volatile than water, and therefore do not condense in the stellar outflow at temperatures $\sim 100-300 \mathrm{~K}$. The $\mathrm{OH}-$ IR spectrum is consistent with the presence of pure $\mathrm{H}_{2} \mathrm{O}$ frosts on silicate cores, whereas the ices in interstellar clouds include molecules involving $C$ and $N$ which condense at $<100 \mathrm{~K}$.

The notable absence of the $11.5 \mu \mathrm{m}$ ice feature in molecular clouds also appears to be related to the impure state of the ices condensing in an interstellar environment. Laboratory investigations have shown that this feature is shifted to longer wavelengths (beyond the edge of the $7-14 \mu \mathrm{m}$ window in the terrestrial atmosphere) when other astrophysically abundant molecules are included in the solid $\mathrm{H}_{2} \mathrm{O}$ matrix (e.g. Tielens and Allamandola, 1987). This provides a natural explanation for its absence in ground-based spectra of molecular clouds. The evolved stars provide the 'control experiment' in which the ice is relatively pure.

\section{CIRCUMSTELLAR EXTINCTION}

The extinction curve of circumstellar dust from near infrared to far ultraviolet wavelengths can, in principle, provide information on both the composition and the size distribution of the particles. Traditionally, interstellar extinction curves are generated by the 'colour difference' or 'pair' method, in which the spectral energy distributions of selected reddened stars (which are generally normal and of early spectral type) are compared with intrinsically similar objects suffering little or no reddening. This technique is much less reliable for circumstellar extinction, 


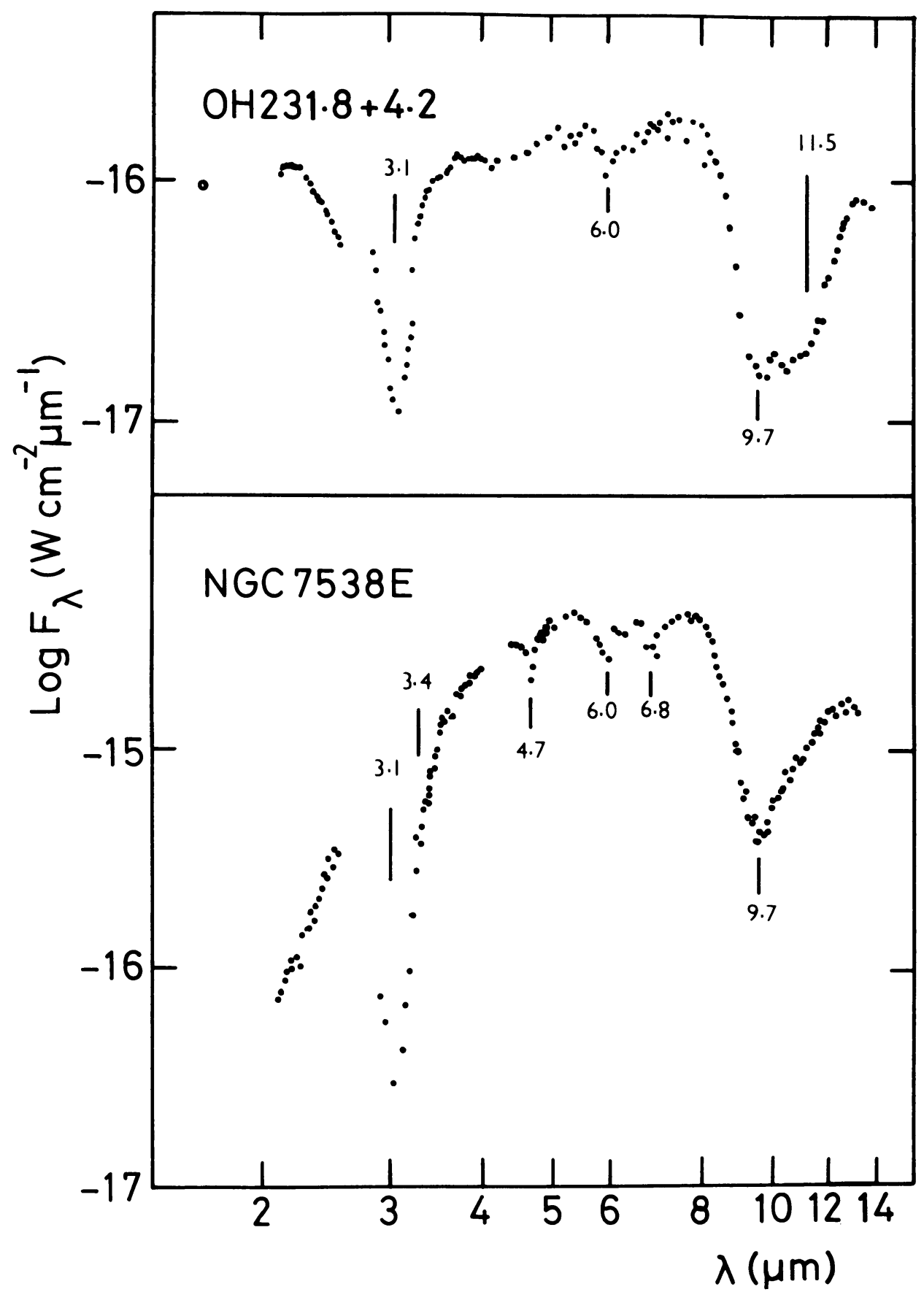

Fig. 4. Infrared spectra of the evolved star OH231.8+4.2 (Soifer et al., 1981) and the molecular cloud source NGC7538E (Willner et al., 1979). Spectral features discussed in the text are labelled in microns. 


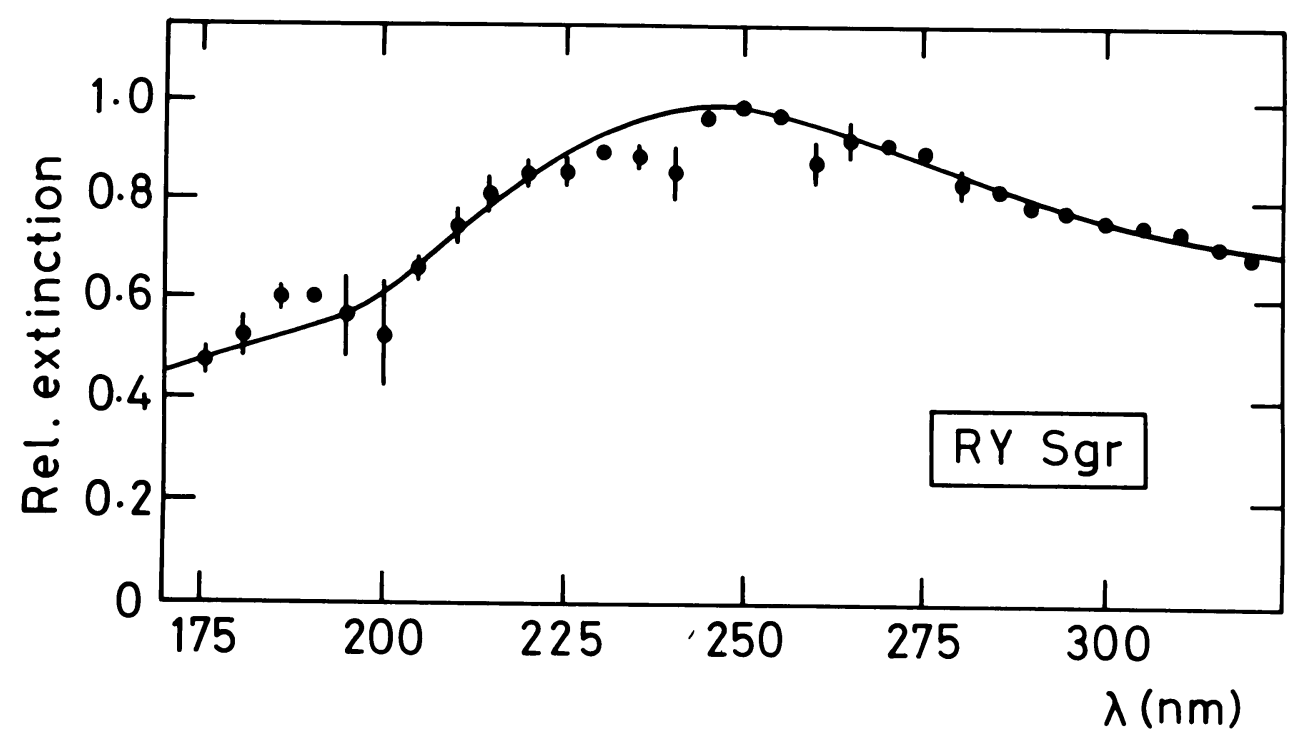

Fig. 5. Circumstellar extinction towards RY Sgr plotted against wavelength, based on IUE data (points). The curve represents the calculated extinction for glassy carbon spheres with radii between 15 and 50nm. (See Hecht et al., 1984).

because in this case the reddened stars are generally intrinsically variable, with complex or anomalous spectra which may include emission lines. Two techniques have been used to alleviate the problem of finding suitable comparisons. Firstly, in cases where stellar variability is caused entirely by varying degrees of circumstellar extinction, the comparison between spectra at bright and faint phases of the light curve provides an extinction curve without reference to another object. Secondly, in serendipitous cases where a normal early-type star lies within or behind a late-type stellar envelope, the former may be used as a probe of the extinction in the latter using the standard technique, provided the interstellar component of the extinction can be reliably corrected for.

Figure 5 shows the circumstellar extinction curve for the RCB-type star RY Sgr, based on IUE data (Hecht et al., 1984). The curve is derived using the variable extinction method, subject to matching the star's pulsational phase at high and low obscuration. The well-known interstellar extinction bump centred at $217 \mathrm{~nm}$ is absent from the circumstellar curve, and replaced by a broad, shallow peak in the 230-250nm region. The latter is characteristic of glassy or amorphous carbon: the data in Figure 5 are consistent with the calculated extinction for glassy carbon spheres of radii 15-50nm which follow a power-law size distribution (Hecht et al., 1984). The absence of $217 \mathrm{~nm}$ absorption has also been reported towards other $C$ rich objects (Buss, Snow, and Lamers, 1989) including the Red Rectangle (Sitko, Savage, and Meade, 1981). The available data are equivocal as to whether the particles responsible for the other prominent feature of the interstellar extinction curve, the far ultraviolet rise, are present in $C$-rich envelopes. 
The circumstellar extinction towards M-type supergiants has been investigated by the 'near star' method (Snow et al., 1987; Buss and Snow, 1988). The data show no evidence for either the bump or the far ultraviolet rise. Towards $\alpha$ Scorpii, for example, the extinction declines steadily with increasing wavelength between 300 and $125 \mathrm{~nm}$. These results are generally consistent with the presence of relatively large (radii $>50 \mathrm{~nm}$ ) siliceous particles in the stellar winds.

\section{CONCLUSION}

Our current knowledge on the composition of dust in stellar ejecta may be summarised as follows.

In $O$-rich stars, the observed grain properties are characteristic of amorphous silicate. This explains the occurrence and shape of the 9.7 and $19 \mu \mathrm{m}$ spectral features, the spectral index of the far infrared emissivity, and the featureless nature of the near to mid-ultraviolet circumstellar extinction curve. It is hard to establish whether silicate material is the main contributor to the total mass of dust in $O$ rich stars generally: if another major component is present, it must be essentially featureless between the wavelengths of $120 \mathrm{~nm}$ and $30 \mu \mathrm{m}$. The only exception is the presence of water frosts in the outflows of a small subset of this stellar group.

In $C$-rich stars, the predominant solid phase is likely to be amorphous carbon. This material can account for both the infrared continuum emission and the midultraviolet extinction. The extended red emission is another observed phenomenon, not discussed here, which is explicable in terms of amorphous carbon (see Duley, 1989). Silicon carbide is also produced in carbon stars, but only accounts for a few percent of the dust mass (see Martin and Rogers, 1987). Crystalline graphite is unlikely to be abundant, but a more extensive search for the predicted graphite feature at $11.5 \mu \mathrm{m}$ should be attempted.

When a $C$-rich red giant evolves to become a planetary nebula, the unidentified infrared emission features become prominent in its spectrum. A major goal for future theoretical work would be to establish the evolutionary link, if any, between amorphous carbon stardust and the carriers of these features (see Frenklach and Feigelson, 1989).

ACKNOWLEDGEMENTS. I should like to thank M. F. Bode for helpful discussions and for access to his collection of reprints on circumstellar dust, and D. Malin for supplying Color Plate 5, which is reproduced courtesy of the Anglo-Australian Telescope Board. Finally, I am indebted to the Royal Society, the IAU, and Lancashire Polytechnic for financial support.

\section{REFERENCES}

Allamandola, L. J., Tielens, A. G. G. M., and Barker, J. R. 1987, in Physical Processes in Interstellar Clouds, eds. G. E. Morfill and M. Scholer, (Dordrecht: Reidel), p. 305.

Bode, M. F. 1988, in Dust in the Universe, eds. M. E. Bailey and D. A. Williams, (Cambridge University Press). 
Bregman, J. 1989, in IAU Symposium 195, Interstellar Dust, eds. L. J. Allamandola and A. G. G. M. Tielens, (Dordrecht: Kluwer), p. 109.

Buss, R. H., and Snow, T. P. 1988, preprint.

Buss, R. H., Snow, T. P., and Lamers, H. J. G. L. M. 1989, in Interstellar Dust Contributed Papers, eds. A. G. G. M. Tielens and L. J. Allamandola, NASA CP-3036.

Cohen, M. 1984, M. N. R. A. S., 206, 137.

Cohen, M., Allamandola, L. J., Tielens, A. G. G. M., Bregman, J., Simpson, J. P., Witteborn, F. C., Wooden, D., and Rank, D. 1986, Ap. J., 302, 737.

de Muizon, M., Geballe, T. R., and d'Hendecourt, L. B. 1986, Ap. J. (Letters), 306, L105.

Draine, B. T. 1984, Ap. J. (Letters), 277, L77.

Duley, W. W. 1989, in IAU Symposium 195, Interstellar Dust, eds. L. J. Allamandola and A. G. G. M. Tielens, (Dordrecht: Kluwer), p. 141.

Dwek, E. 1989 , in IAU Symposium 195, Interstellar Dust, ed. L. J. Allamandola and A. G. G. M. Tielens, (Dordrecht: Kluwer), p. 479.

Eiora, C., Hefele, H., and Zhong-yu, Q. 1983, Astr. Ap. Suppl. Ser., 54, 309.

Frenklach, M., and Feigelson, E. D. 1989, Ap. J., preprint.

Geballe, T. R., Kim, Y. H., Knacke, R. F., and Noll, K. S. 1988, Ap. J. (Letters), 326, L65.

Gehrz, R. D. 1989, in IAU' Symposium 195, Interstellar Dust, ed. L. J. Allamandola and A. G. G. M. Tielens, (Dordrecht: Kluwer), p. 445.

Gehrz, R. D., Ney, E. P., Grasdalen, G. L., Hackwell, J. A., and Thronson, H. A. 1984, Ap. J., $281,303$.

Gehrz, R. D., Grasdalen, G. L., Greenhouse, M., Hackwell, J. A., Heyward, T., and Bentley, A. F. 1986, Ap. J. (Letters), 308, L63.

Glasse, A. C. H., Towlson, W. A., Aitken, D. K., and Roche, P. F. 1986, M. N. R. A. S., $220,185$.

Goebel, J. H., and Moseley, S. H. 1985, A p. J.' (Letters), 290 , L35.

Hecht, J. H., Holm, A. V., Donn, B., and Wu, C. C. 1984, Ap. J., 280, 228.

Hildebrand, R. H. 1983, Quart. J. R. A. S., 24, 267.

Humphreys, R. M., and Ney, E. P. 1974, Astr. Ap., 30, 159.

Le Bertre, T. 1987, Astr. Ap., 176, 107.

Little-Marenin, I. R. 1986, Ap. J. (Letters), 307, L15.

Martin, P. G., and Rogers, C. 1987, Ap. J., 322, 374.

Nuth, J. A., Donn, B., and Nelson, R. 1986, Ap. J. (Letters), 310, L83.

Papoular, R., and Pegourie, B. 1983, Astr. Ap., 128, 335.

Roche, P. F. 1988, in IAU Symposium 191, Planetary Nebulae, ed. S. Torres-Peimbert, (Dordrecht: Reidel).

Roche, P. F., and Aitken, D. K. 1984a, M. N. R. A. S., 208, 481.

Rocher. $1984, M . N . R . A . S .209,33$ P.

Rowan-Robinson, M., Lock, T. D., Walker, D. W., and Harris, S. 1986. M. N. R. A. S., 222, 273.

Salpeter, E. E. 1977, Ann. Rev. Astr. Ap., 15, 267.

Sedlmayr, E. 1989, in IAU Symposium 195, Interstellar Dust, eds. L. J. Allamandola and A. G. G. M. Tielens, (Dordrecht: Kluwer), p. 467.

Simpson, J. P., and Rubin, R. H. 1989, in Interstellar Dust Contributed Papers, eds. A. G. G. M. Tielens and L. J. Allamandola, NASA CP-3036.

Sitko, M. L., Savage, B. D., and Meade, M. R. 1981, Ap. J., 246, 161.

Snijders, M. A. J., Batt, T. J., Roche, P. F., Seaton, M. J., Morton, D. C., Spoelstra, T. A. T., and Blades, J. C. $1987, M$. N. R. A. S., 228, 329.

Snow, T. P., Buss, R. H., Gilra, D. P., and Swings, J. P. 1987, Ap. J., $321,921$.

Soifer, B. T., Willner, S.' P., Capps, R. W., and Rudy, R. J. 1981, Ap. J., 250, 631.

Sopka, R. J., Hildebrand, R. H., Jaffe, D. T., Gatley, I., Roellig, T., Werner, M., Jura, M., and Zuckerman, B. 1985, Ap. J., 294, 242.

Tielens, A. G. G. M., and Allamandola, L. J. 1987, in Physical Processes in Interstellar Clouds, eds. G. E. Morfill and M. Scholer, (Dordrecht: Reidel), p. 333.

Tokunaga, A. T., Nagata, T., Sellgren, K., Smith, R. G., Onaka, T., Nakada, Y., Sakata, A., and Wada, S. 1988, Ap. J., 328, 709 .

Tokunaga, A. T., Sellgren, K., Sakata, A., Wada, S., Onaka, T., Nakada, Y., and Nagata, T. 1989, in Interstellar Dust Contributed Papers, eds. A. G. G. M. Tielens and L. J. Allamandola, NASA CP-3036.

Treffers, R. R., and Cohen, M. 1974, Ap. J., 188, 545.

Whittet, D. C. B. 1988, in Dust in the Universe, ed. M. E. Bailey and D. A. Williams, (Cambridge University Press).

Willner, S. P, Puetter, R. C., Russell, R. W., and Soifer, B. T. 1979, Ap. Space Sci., 65, 95.

Witt, A. N. 1985, Ap. J., 294, 216. 